\title{
Acute Massive Aortic Dissection Secondary to Vascular Endothelial Growth Factor Inhibitor: An Uncommon Presentation of Drug-Induced Cardiovascular Toxicity
}

\author{
Joey Saliba L MD ${ }^{1 *}$, Jasneet Devgun Do ${ }^{1}$ \\ ${ }^{1}$ The Ohio State University- Wexner Medical Center, Department of Internal Medicine
}

${ }^{\star}$ Corresponding Author: Dr. Joey Saliba L mD, Department of Internal Medicine, The Ohio State University- Wexner Medical Center, USA; E-mail: lawrence.saliba@ osumc.edu

Received: January 29, 2020; Accepted: February 06, 2020; Published: February 13, 2020

\section{Introduction}

In a patient presenting with severe acute chest or back pain, aortic dissection is a concerning etiology and requires immediate attention due to its profound morbidity and mortality. Less common presentations include altered mental status, stroke symptoms, and syncope, which represent less than $30 \%$ of aortic dissections. Risk factors have been well described, and include history of uncontrolled hypertension, connective tissue disease, and vasculitis. We present a case of acute Stanford Type A aortic dissection in a patient without risk factors but with recent history of bevacizumab treatment, who also presented with atypical symptoms.

\section{Case Description}

The patient is a 60 year old female with past medical history of colon cancer metastatic to liver and lung, who woke from sleep with severe chest pain, followed by right facial droop and right upper extremity weakness. Stroke code was called and Glasgow Coma Scale was 5 on admission, but CT Head was negative for hemorrhage or large-territory infarct. CT angiography of the brain and neck partially showed a Stanford type A aortic dissection, and CT aneurysm study confirmed the dissection, which extended from the aortic valve to the femoral arteries and included the great vessels, celiac trunk, and superior mesenteric artery. She was in shock on admission, was intubated and sedated. Based on the patient's frailty she was a poor surgical candidate, and the family stated she would have declined repair. She lived for another day in the ICU before transitioning to comfort care.

Postmortem review revealed no classic risk factors for aortic dissection. The patient had no history of hypertension, connective tissue disease, or vasculitis, prompting further evaluation of her extensive history of chemotherapy. She was originally diagnosed with moderately-differentiated colon cancer in 2012 and subsequently underwent multiple resections of the primary tumor and metastasis (liver and peritoneum). Bilateral pulmonary nodules were found and treated medically. Multiple antitumor agents were used since 2012, the majority of which involved many iterations of fluorouracil and bevacizumab. The last chemotherapy regimen consisted of FOLFIRI and bevacizumab, which was resumed four months prior to the dissection.

\section{Discussion}

This case illustrates the broad range of presentations of aortic dissection, including altered mental status or stroke symptoms, as well as devastating complications of certain antitumor regimens. Cardiovascular side effects can be the most profound, especially in the use of fluoropyrimidines (e.g. fluorouracil) and vascular endothelial growth factor (VEGF) pathway inhibitors (e.g. bevacizumab). In the absence of typical risk factors, repetitive vascular toxicity from these agents likely represents the causative pathophysiology for the acute dissection. While aortic dissection is an uncommon complication of these therapies, it is incumbent on providers to recognize this possibility and recall the variable presentations of the pathology.

\section{Citation:}

Joey Saliba L, Jasneet Devgun (2020) Acute Massive Aortic Dissection Secondary to Vascular Endothelial Growth Factor Inhibitor: An Uncommon Presentation of Drug-Induced Cardiovascular Toxicity. J Cardiol Clin Pract, Volume 3(1): 1-2. 


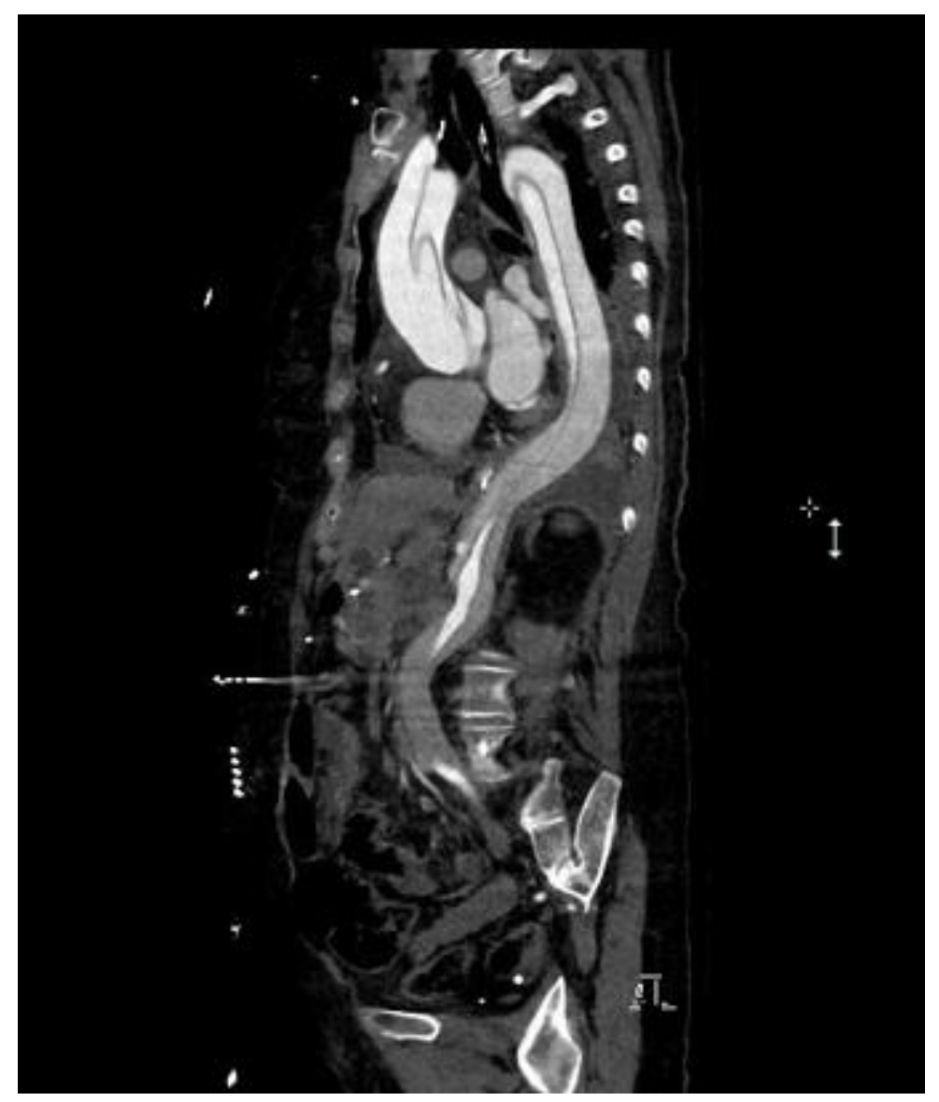

Figure 1. Sagittal view showing Stanford type A aortic dissection extending from aortic valve into abdomen

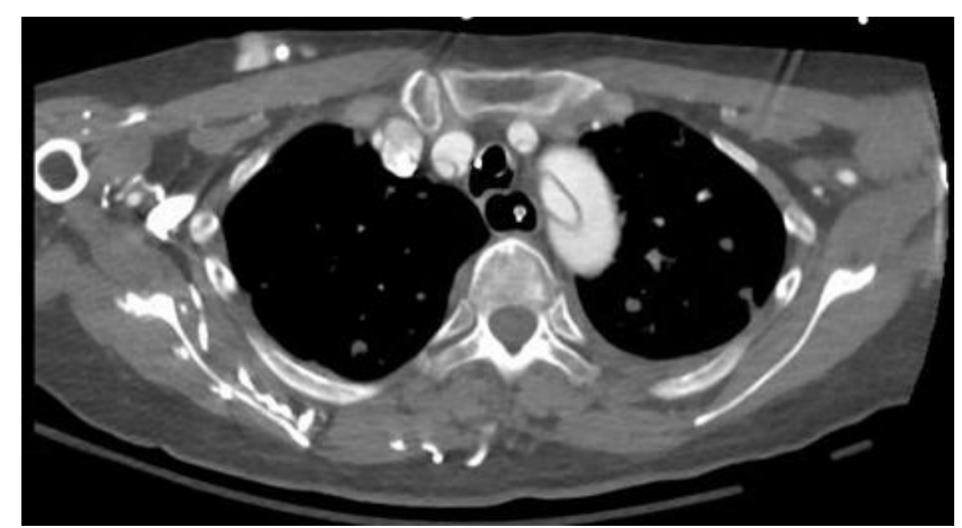

Figure 2. Axial view showing aortic dissection involving arch and all three arch arterial branches. 